\title{
MODIFICACIONES DEL PERFIL DE PLAYAS EN LAS ISLAS BALEARES (PLAYAS DE CAN PICAFORT Y ES COMÚ DE MURO)
}

\author{
Miquel Mir-Gual \\ Departamento de Ciencias de la Tierra \\ Universidad de las Islas Baleares
}

\section{RESUMEN}

La situación económica y social en la isla de Mallorca hace que los espacios litorales, con énfasis sobre las costas arenosas, sean considerados a partir de su rentabilidad y en ningún caso a partir de sus condiciones ambientales, variables y vulnerables. Eso hace que éstos sean espacios sobreexplotados en temporada alta, en detrimento a su percepción a lo largo de los meses invernales, pasando a ser espacios olvidados. El presente trabajo pretende mostrar así el dinamismo que estos lugares proyectan en una escala temporal estacional, y de los distintos comportamientos mostrados en dos playas diferentes, dependiendo de su grado de antropización y frecuentación.

Palabras clave: Playa urbana, playa natural, variabilidad estacional, medidas de gestión.

\section{ABSTRACT}

Mallorca's economic and social situation makes that the coast area, with emphasis in sandy coasts, has been considered from its profitability and in any case from their environmental conditions, changeables and vulnerables. The perception exposed cause that this space is crowded along the high season (summer), against the perception have it along the low season (winter), becoming forgotten spaces. In this way, the present paper expect to show and prove the dynamism that these places extend along a seasonal scale, and about the different behaviours showed by the beaches studied, depending of the human impacts.

Key words: Urban beaches, natural beaches, season variability, management. 


\section{Introducción}

Hoy en día es un hecho cotidiano hablar de litoral, sobre todo en un emplazamiento turístico como es el mar Mediterráneo, en donde gran parte de su actividad económica se desarrolla sobre su franja costera. No obstante, la percepción que uno tenga del espacio litoral puede discrepar de otros puntos de vista. Las interpretaciones que hoy se hacen de la costa son realmente heterogéneas, respondiendo siempre a los intereses de distintos colectivos. Sea como sea, en el lenguaje coloquial aparecen recurrentemente términos tales como litoral o costa, a pesar de que en la literatura científica no aparezca la precisión para definirlos (Suárez de Vivero, 1999). Es cierto pero, que dentro de este abanico, la imagen mental más frecuente sobre este espacio se traduce en verlo e interpretarlo como recurso turístico y/o económico, todo y la perceptible peligrosidad que se le podría atribuir a éste, consecuencia de los recurrentes episodios naturales que en él tienen lugar (Bauer y Sherman, 1999).

La interacción de factores ambientales en el ámbito costero determina en él una gran variedad de procesos. La consecuencia de este hecho se traduce en que la franja litoral sea hoy considerada como una de las zonas naturales más frágiles y vulnerables del planeta (Hernández Calvento, 2002). La ocupación y asentamiento de la actividad humana, y el crecimiento exponencial de actividades y usos ajenos a estos emplazamientos, han hecho que hoy, gran parte de la zona costera, con predominancia en las costas bajas arenosas, se haya visto gravemente afectada. A modo de aproximación, y según postula Nonn (1987), el $20 \%$ de las costas a nivel mundial son de génesis arenosa, de las cuales el $70 \%$ se erosionan, bien por causas naturales o bien por la acción destructiva de la actividad humana.

Los continuos atentados contra el medio físico-natural palpables hoy en muchas zonas litorales, han demostrado a lo largo del tiempo que son sintomáticos de una falta crónica de integración transversal en su gestión (OCDE, 1995). La pretensión de las economías en contabilizar sus éxitos a través de «pseudo medidas monetarias», dificulta cada vez más contemplar la realidad natural, calculada basándose en el sistema métrico decimal (Murray, 2005), en detrimento a la consideración de sus características y potencialidades ambientales y ecológicas.

La isla de Mallorca, y el comportamiento de sus playas a escala anual, son un buen indicador de lo dicho. El monopolio que la industria turística lleva teniendo sobre la franja litoral desde mediados de la pasada centuria hace, temporada tras temporada, que la consideración de las playas sólo se enfoque hacia los meses de verano, siendo espacios olvidados a lo largo del período invernal. Las consecuencias derivadas de esto, hacen que las medidas de gestión en dichos espacios sean intensas de cara a la temporada turística, y prácticamente ausentes el resto del año, provocando fuertes impactos, en muchos casos irreversibles.

\section{Contextualización de la zona}

Las playas estudiadas se localizan en la bahía de Alcúdia, en el cuadrante nororiental de la isla de Mallorca (Figura 1). Las características biogeográficas de la misma bahía han ayudado al desarrollo de un gran número de sistemas dunares que, según Servera (1997), se podían considerar como un único sistema hasta su fragmentación. Ésta fue iniciada a mediados del siglo pasado, consecuencia del masivo y descontrolado desarrollo urbanístico. $\mathrm{Y}$ es precisamente esa tendencia la que hoy tenemos que buscar para dar explicación a las diferencias existentes entre las playas objeto de estudio que, a pesar de su contigüidad, se caracterizan de manera notablemente diferente. 


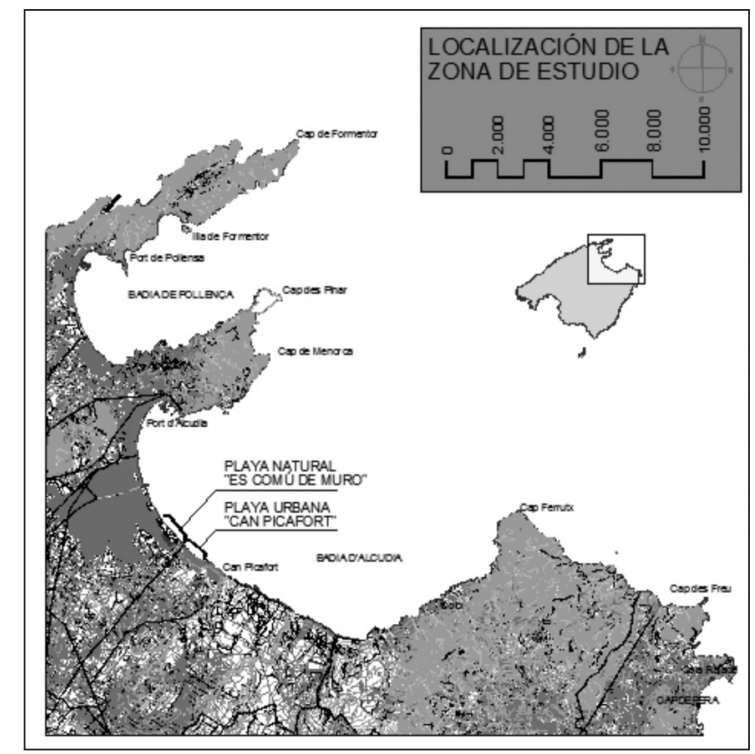

FIgURA 1. Localización de la zona de estudio.

Las diferentes intensidades de presión antrópica, así como la protección de la franja de dunas de Es Comú de Muro en 1988, con la declaración del Parque Natural de s’Albufera de Mallorca, han hecho que hoy las características fisonómicas de las playas estudiadas disciernan en algunos aspectos. El núcleo turístico de Can Picafort, perteneciente al municipio de Santa Margalida, representa uno de los puntos turísticos por excelencia en la isla. Éste, desde 1960, entonces un pequeño núcleo de pescadores, lleva incrementando su oferta turística interrumpidamente (Tabla 1).

Tabla 1

EVOLUCIÓN DE LAS PLAZAS HOTELERAS EN EL NÚCLEO DE CAN PICAFORT (SCHÜRGERS, 2007)

\begin{tabular}{|c|c|c|c|c|}
\hline Año & $\mathbf{1 9 6 3}$ & $\mathbf{1 9 7 2}$ & $\mathbf{1 9 8 1}$ & $\mathbf{1 9 9 2}$ \\
\hline $\mathbf{N}^{\mathbf{0}}$ plazas hoteleras & 1.400 & 8.750 & 9.274 & 9.448 \\
\hline
\end{tabular}

El resultado del proceso ha sido un crecimiento urbanístico anárquico, sin ningún tipo de control ni planeamiento (Mas, 1992), dando como producto un emplazamiento turístico de primer orden a escala insular. Éste supone en la actualidad una presión antrópica materializada en 12.865 plazas turísticas (Schürgers, 2007), además de una oferta complementaria no menos importante, y la construcción de un puerto deportivo y un paseo marítimo. Con este escenario, la primera playa estudiada adopta una fisonomía plenamente urbana. Con un frente aproximado a 1,5 kilómetros, y velada por la primera línea hotelera, la playa de Can Picafort es hoy un 
paradigma de playa antropizada, con cantidad de servicios enfocados a los usuarios, y con serios problemas en temporada baja frente a los temporales de mar, frecuentes en tormentas de dirección E-NE, como ya quedó demostrado en noviembre de 2001, que con un fuerte temporal de mar, la playa desapareció debido a la erosión, provocando a una regeneración artificial en 2002, con sedimento de características divergentes a las naturales, extraído de la costa de Banyalbufar, localizado al noroeste de la isla. (Basterretxea et al., 2002).

Limítrofe con la anterior, encontramos la playa natural de Es Comú de Muro, inserida dentro de los límites protegidos por el Parque Natural de s'Albufera de Mallorca. El hecho de estar ésta sometida a una figura de protección, no ha sido sinónimo de liberación frente a la presión del hombre. Su localización, haciendo frontera con dos grandes núcleos turísticos (las playas de Muro y Can Picafort), supone que este lugar sea altamente frecuentado por los turistas y residentes en la zona, suponiendo alteraciones de calado para el sistema natural. La playa de Es Comú, con un frente que se sitúa alrededor de los 1,8 kilómetros, se caracteriza por ser una playa natural, de arenas finas y medias, y con un $89 \%$ de composición bioclástica (Servera, 2002). Forma parte del sistema playa-duna de Es Comú, el cual nos permite definir, por sus características biológicas y geomorfológicas, el modelo que siguen estos ambientes en el resto de las Islas Baleares (Gelabert et al., 2002). Actualmente los signos de degradación que se presentan, sobretodo en la primera línea de foredunes, son de significativa importancia, dibujando un sistema altamente fragmentado con un notable número de geoformas de deflación en su frente.

\subsection{Impactos en la zona de estudio y gestión actual}

Sin duda, el desarrollo urbanístico ha sido el principal agente en tanto a los impactos que podemos determinar en las zonas que nos ocupan, y responsable a la vez de los desequilibrios dinámicos que soporta el sistema playa-duna (Goy et al. 1997). La transformación territorial vivida a lo largo de las últimas décadas no ha sido en vano para el medio físico de la zona, sino que de él han derivado actuaciones irreversibles (Mas, 1992). En el caso de la playa de Can Picafort, el resultado no ha sido otro que la destrucción de buena parte del sistema dunar (Figura 2), artificializando por completo la parte emergida del sistema e interfiriendo su dinámica natural.

A parte de los efectos in situ, algunas de las actuaciones en la playa de Can Picafort también han afectado indirectamente la franja natural de Es Comú. Las distintas regeneraciones artificiales de sedimento llevadas a cabo en la zona a lo largo de las últimas décadas, han supuesto cambios en la morfología del tamaño de grano, ya que con una deriva litoral con dirección S-N, el sedimento artificial ha sido transportado hacía la franja natural (Servera, 2004; Basterretxea et al., 2007).

El creciente desarrollo económico no se ha visto limitado a la zona terrestre, sino que también ha afectado la parte submarina, con la presencia de puertos deportivos, obras hidráulicas de magnitudes importantes, e infraestructuras de abrigo, generando cambios importantes en la dinámica natural de los sistemas playa-duna (Servera, 1997), y postulándose como trampas sedimentarias de calado que han obligado a un nuevo reajuste de la línea de costa desde su aparición (Martín-Prieto y Servera, 2006).

Precisamente, los problemas que hoy afloran en este lugar, al igual que en otros muchos espacios litorales de la isla, son consecuencia directa de la mala gestión, o en muchos casos, inexistente. Cuando hablamos de la gestión que se viene practicando en las playas objeto de estudio nos referimos principalmente a las técnicas de limpieza que se utilizan en ellas. Desde decenios atrás, la gestión de las playas ha estado marcada por la preocupación de cumplir con las normas básicas de higiene y por la instalación de ciertos servicios destinados al ocio y recrea- 
ción de usuarios, no concibiendo estos espacios como elementos naturales, frágiles y dinámicos (Roig-Munar, 2004). La alta rentabilidad de las playas, en el caso de la playa de Benidorm traducida en $12.000 \square /$ año/m² según Yepes (2002), ha hecho que la voz de los empresarios turísticos haya sido la de mayor ocurrencia sobre estos espacios, luchando año tras año para mantener playas extensas, de arena fina y blanca, y sin restos vegetales que aparenten suciedad.

El hecho de hacer funcionales los espacios litorales para satisfacer su uso, ha traído consigo serios problemas de conservación y estabilidad de los sistemas litorales (RoigMunar, 2003), que no tan sólo son palpables en un período medio-largo de tiempo, sino que pueden aparecer a escala anual.

\section{Objetivos, materiales y métodos}

\subsection{Objetivos}

Se postula que uno de los primeros pasos que se deberían de dar para la correcta gestión de playas pasa por el cambio de la percepción que sobre estos espacios tiene la sociedad en general, pero sobre todo los agentes gestores que influencian sobre él. Partiendo de esta premisa se estructura el presente trabajo. Las playas no deben ser vistas simplemente como un espacio generador de divisas, sino que ante todo se deben considerar por su vulnerabilidad y complejidad como sistema. Tal y como ya han postulado algunos autores en diferentes estudios, y partiendo de las divergentes características entre playas (Juaneda y Roig-Munar, 2002; Roig-Munar, 2003; Roig-Munar y Comas-Lamarca, 2005), el conocimiento transversal de estos espacios debería de ser el pilar sobre el cual pivotara la gestión y las actuaciones en estos ambientes.

Las playas arenosas en las Baleares, al igual que ocurre en las playas estudiadas, se presentan como espacios bipolares en cuanto a su utilización y percepción. Así, la funcionalidad litoral se limita a los meses de verano, mirando hacía los intereses turísticos, y sobreexplotando sus capacidades naturales. Si bien algunas actuaciones ya hechas hoy asumen un estado irreversible, la gestión actual debería asumir una visión de más perspectiva, abordando mayor transversalidad. Nos referimos así no sólo a las técnicas utilizadas, sino también a la consideración del espacio «playa» a escala temporal.

De esta reflexión nace el objetivo central del presente estudio. Del trabajo de campo se ha obtenido información cualitativa y cuantitativa que nos permite demostrar la variabilidad de las playas estudiadas a lo largo de un año. Así, de la valoración e interpretación de las diferentes variables mostradas, algunos son los resultados merecedores de análisis.

\subsection{Materiales y métodos}

Para ello se han realizado 6 perfiles a lo largo de la playa urbana de Can Picafort y la playa natural de Es Comú de Muro, perteneciente al Parque Natural de s'Albufera de Mallorca. Del total, dos de ellos pertenecen a la playa urbana, uno a la zona de transición, y los tres restantes se sitúan ya dentro de la franja natural.

La determinación y geolocalización de los perfiles se ha llevado a cabo a través de GPS, modelo GARMIN Dakota 20 de alta sensibilidad, a fin de localizar con precisión el punto de referencia para cada uno de los perfiles, y así conseguir el mismo nodo de partida en cada uno de los días de muestreo. El trabajo de campo se ha llevado a cabo a lo largo de un año, con una recurrencia aproximada a los dos meses, con el fin de tomar perfiles en las diferentes estaciones. Así se presentan datos tomados en los días 13/6/09, 27/8/09, 27/10/09, 30/1/10, 27/3/10, 29/5/10. 

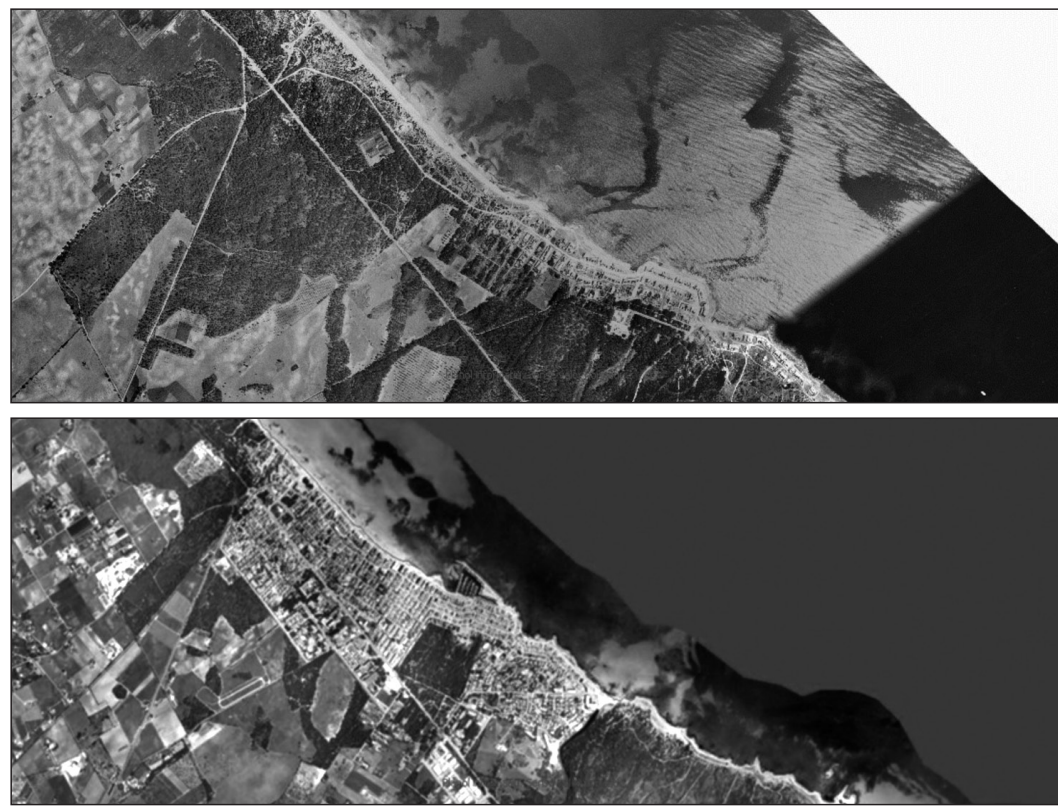

Figura 2. Fotografias aèreas de Can Picafort (1956-2008).
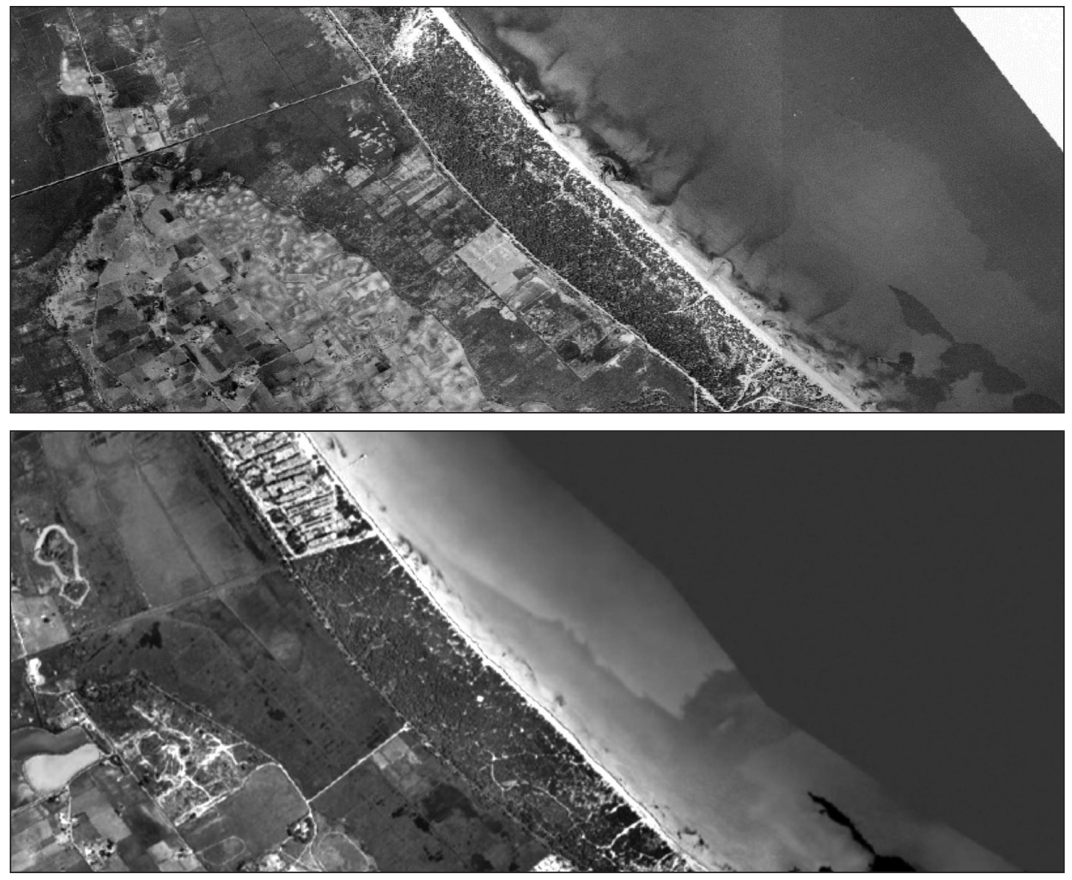

Figura 2. Fotografias aèreas de Es Comú de Muro (1956-2008).

Fuente: Infraestructura de dades espacials de les IB (www.ideib.cat). 
Las mediciones de la playa, y tomando como referencia las teorías defendidas por Emery (1961), Delgado y Loid (2004) y Puleo et al. (2008), se han realizado con un pantómetro de 1,5 x 1,5 metros (Figura 4), con el objetivo de obtener información para trazar perfiles bidimensionales con ejes de longitud (x) y altura (y). El punto de referencia tomado ha sido el inicio de cada uno de los perfiles, debido a su poca variabilidad (inexistente en el caso de la playa urbana), y al gran dinamismo del otro posible punto de referencia, la zona de swash. La toma de datos para cada uno de los perfiles aborda toda la superficie del backshore, así como los primeros metros del nearshore, dependiendo de la existencia o no de barras sumergidas y de su distancia respeto a la zona de swash. La información in situ fue anotada en fichas de campo, en donde se constataban variables cualitativas de los días respectivos, tales como la existencia de bermas de Posidonia oceanica, la existencia de ripples, de marcas de temporales en el backshore, la distancia entre el punto de referencia y el swash, la presencia o ausencia de barras submergidas, o la existencia de escarpes arenosos en la playa alta, entre otras.

Para la medición de los grados de pendiente se ha utilizado un calculador de ángulos de alta precisión, modelo Tajima SLANT 100, con alta capacidad para establecer y medir ángulos e inclinaciones.

Posteriormente, la información tomada en el campo ha sido procesada bajo los principios de Pitágoras y en la medida que Puleo et al. (2008) indican. Así, la hipotenusa se fija en 1,5 metros (distancia entre las barras del pantómetro), mientras que el eje vertical del triángulo es la medida tomada menos el nivel de la superficie plana $\left(y-y_{L}\right)$, y el eje horizontal viene representado por la distancia entre la medida actual $\left(\mathrm{X}_{\mathrm{i}+1}\right)$ y la previa tomada $\left(\mathrm{X}_{\mathrm{i}}\right)$.

$$
X_{i+1} X_{i}=\sqrt{1-\left(y-y_{L}\right)^{2}}
$$

Del resultado gráfico, junto con las fichas de campo rellenadas, las variables calculadas se expresan en:

- Variación de la pendiente para cada perfil, entre el mínimo y el máximo de la secuencia.

- La existencia de barras sumergidas y su distancia con la zona d'swash.

- La existencia de bermas de Posidonia oceanica y su distancia con el swash.

- Existencia de restos de temporales en el backshore.

- Variación longitudinal del perfil, tomando como referencia el punto d'swash.

- Volumen sedimentario por perfiles y días de muestreo.

Para el cálculo de los volúmenes de arena presentados, y partiendo de la línea base de referencia determinada, se calcula la superficie de la sección por tramos verticales, cada uno de ellos respondiendo a la suma de un rectángulo inferior más su triángulo superior (para completar la superficie que resta hasta la línea dibujada por el perfil calculado) (Figura 3).

La superficie de cada triangulo se obtiene por la diferencia entre la base (x) multiplicada por la altura (y), dividiéndolo entre 2,

$$
\Delta \mathrm{X} * \Delta \mathrm{Y} / 2
$$

mientras que en el caso de los rectángulos, se miden multiplicando la base del rectángulo (x) por la altura (a) del rectángulo anterior, menos $\Delta \mathrm{Y}$. 


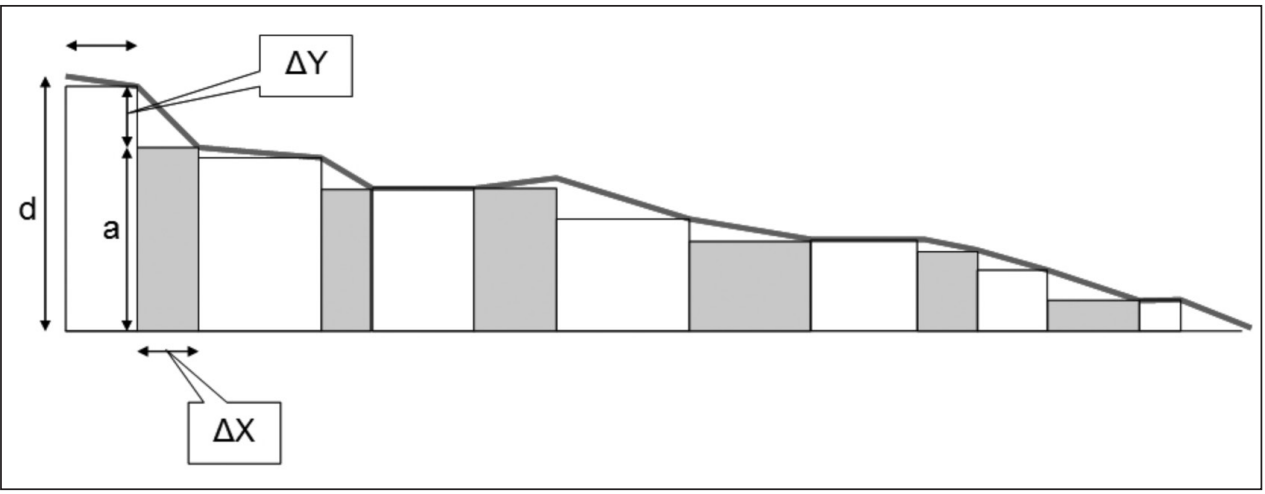

FIGURA 3. Representación gráfica de la metodología utilizada para cálculo de volúmenes.

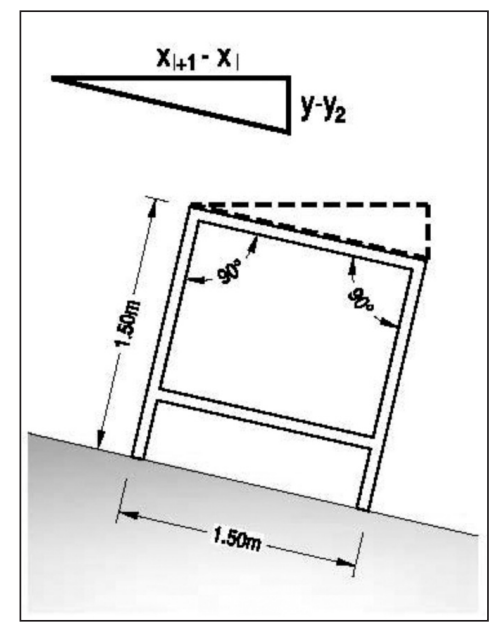

FIgURA 4. Representación gráfica de la metodología utilizada en los perfiles de playa.

$$
(\Delta \mathrm{X} * \mathrm{a})-\Delta
$$

A fines comparativos, en algunos casos se ha procedido al análisis de la relación lineal entre variables cuantitativa a través del coeficiente de correlación de Pearson,

$$
r=\frac{\sigma_{X Y}}{\sigma_{X} \cdot \sigma_{Y}}
$$

en donde $\sigma_{X Y}$ es la covarianza de $(X, Y)$ y $\sigma_{X}$ y $\sigma_{Y}$ las desviaciones típicas de las distribuciones marginales. 


\section{Resultados y discusión}

La variabilidad de las playas es sin duda su rasgo más característico. Éstas no se mantienen fijas en una posición, sino que cambian su perfil, configuración y planta, a lo largo del tiempo. Al ser espacios tan dinámicos, en donde gran número de factores y agentes se dan lugar, la facilidad y vulnerabilidad frente al cambio morfológico queda justificada. No obstante, estos espacios también vienen modificados por la acción y el comportamiento de sus usuarios. Así no es difícil establecer algunas diferencias entre los patrones de variabilidad de playas urbanas y naturales, en donde los parámetros de frecuentación y presión siempre son distintos.

Se pretende demostrar a continuación, contribuyendo así con la verificación de lo dicho, que esta variabilidad no se manifiesta en períodos largos de tiempo, sino que se hace patente en series mensuales o estacionales. Además de la dimensión temporal, la complejidad del litoral hace de él un espacio con capacidad de cambios en una escala espacial relativamente pequeña. Así vemos como en el caso estudiado, los patrones seguidos en dos playas totalmente limítrofes pueden discernir substancialmente.

Se puede constatar que la línea de costa comprendida en los sectores de Can Picafort y Es Comú de Muro ha recibido variaciones importantes a lo largo del último año (Figura 6). No obstante, y como ya se remarcaba en el párrafo anterior, las diferencias entre el sector natural y urbano son notablemente apreciables. En términos generales, la variabilidad de la playa urbana ha sido considerablemente más elevada que en el caso de los sectores menos antropizados. Los patrones de frecuentación y las técnicas utilizadas para gestionar la playa emergida, suscitan una alta correlación en tanto a la modificación de los perfiles de playa. La zona comprendida entre los perfiles 1 y 3 , perteneciente a la playa urbana, es la de mayor variabilidad, mientras que su prolongación hacia el norte dibuja un trazado de líneas más homogéneas, reflejando el comportamiento que se ha registrado en la zona central de Es Comú. El retorno a la heterogeneidad en el sector norte de la playa de Es Comú responde otra vez a causas antrópicas. El hecho de coincidir con una de las principales entradas a la playa, una zona de parking y el límite de la planta hotelera de las playas de Muro, hace que la tendencia de variabilidad repita los patrones seguidos en la zona urbana. Para más precisión en lo dicho, la variabilidad de perfiles respeto a la mediana $(33,67 \mathrm{~m})$ confirma la tesis expuesta. De esta manera, las medias individuales de los perfiles 1 (38,33 m), $2(37,33 \mathrm{~m})$ y $3(43,92 \mathrm{~m})$ son los que disciernen más de la media global, mientras que en la zona central de Es Comú, en el perfil $5(19 \mathrm{~m})$, la variabilidad disminuye, incluso por debajo de la mediana.

Los cambios en tanto a la posición de la línea de costa también asumen diferencias a escala temporal. Según los muestreos tomados, las máximas longitudes en la playa alta se dan en los meses invernales, de temporada baja, momento en que la frecuentación y uso de la playa es de menor intensidad. Así se ve como las medianas más altas las encontramos en los meses de enero $(40 \mathrm{~m})$, marzo $(38,5 \mathrm{~m})$ y mayo $(35,5 \mathrm{~m})$, mientras que las mínimas se alcanzan en los meses en que el uso y la explotación de la playa es más elevado, junio (31 m), agosto $(28,83 \mathrm{~m})$ y octubre $(28,42 \mathrm{~m})$ (Figura 7$)$.

Algunos son los factores que potencian esas tendencias. En temporada invernal, las acumulaciones de Posidonia oceanica en playa alta instan al crecimiento substancial de la parte emergida, hecho que supone un aumento de su longitud. Así, se percibe como en enero, momento en que las banquetas de $P$. oceanica asumen su máximo esplendor, el crecimiento de la playa alta es patente, alargando su longitud y dotando de unas estructuras de defensa la playa emergida frente a temporales de oleaje. En detrimento de ello, la retirada de esta fanerógama en vistas a la temporada turística acelera la desprotección de la playa consecuencia de la energía refractada por las olas, que junto a la alteración provocada por el uso masivo del espacio, supone un retroceso de la línea de costa y disminución de su espacio utilizable (Figura 5). 


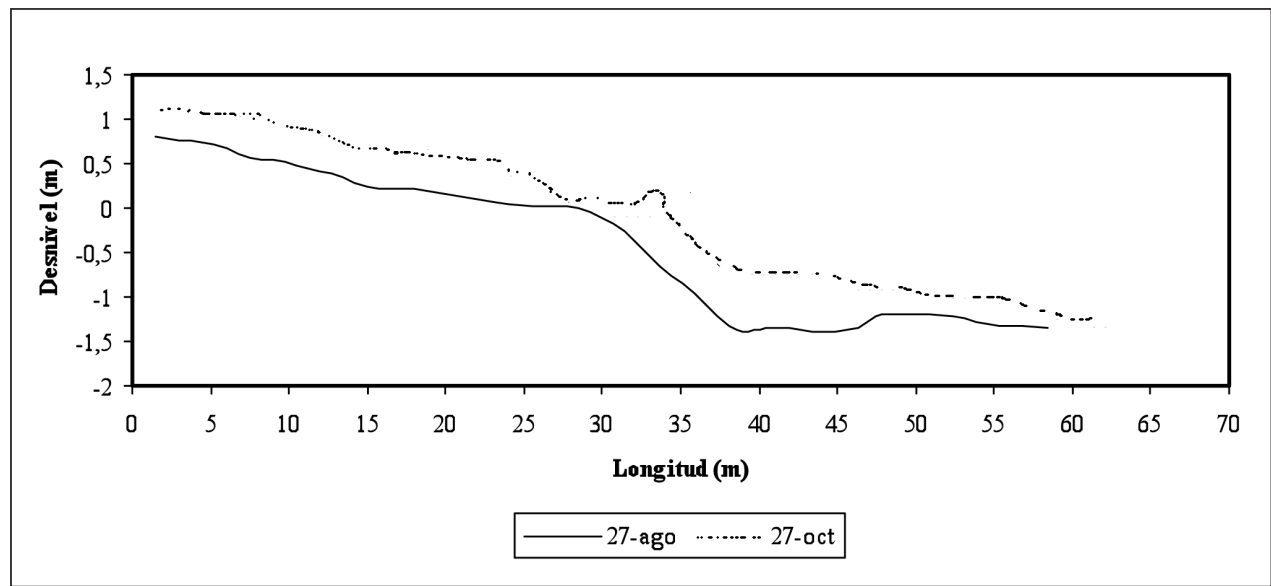

FIgURA 5. Efectos morfológicos de las bermas de Posidonia oceanica en el backshore. Elaboración propia.

La variabilidad de los perfiles de playa medidos no tan sólo se detecta en su longitud, sino que también en su desnivel. Tomando como referencia inicial las medianas para cada uno de los perfiles a lo largo del tiempo de muestreo, dos asociaciones son las que se muestran representativas ya en primera instancia (Figura 8). En primer lugar, la correlación entre el perfil 1 y 2 es casi perfecta, con un trazado de playa muy similar, y con un desnivel medio que se aproxima a los $-4 \mathrm{~m}$. Los dos trazados, siendo los únicos que tienen como punto de referencia el paseo marítimo, dibujan un perfil suave y con pocas alteraciones. En segunda instancia, se ve una nueva asociación formada por el resto de perfiles, más heterogénea y con unos desniveles que oscilan entre los -4 y $-6 \mathrm{~m}$. Dentro de este último grupo, se percibe una diferencia entre los perfiles 5 y 6 , localizados de lleno en la franja más natural de la playa, y los perfiles 3 y 4 , asociados a la zona de transición entre los dos sectores. A pesar de que las diferencias no son muy de calado, éstas se manifiestan sólo en la parte emergida de la playa, ya que como se puede observar, la correlación en la zona de swash y nearshore es palpable.

Las variaciones longitudinales y verticales de perfiles llevan consigo cambios en el depósito de sedimento de la playa. La variabilidad de pendientes, así como también la dinámica del oleaje y transporte litoral, hacen que los volúmenes de arena vayan manifestando cambios significativos. Más que diferenciándolos entre perfiles, esos cambios se presentan significativos si son considerados temporalmente, determinando su caracterización en función de su estacionalidad. Puesto que no siempre se ha tomado la misma longitud en cada uno de los días de muestreo, la playa emergida será la referencia utilizada. Los cambios longitudinales en los perfiles estudiados son los que determinan en mayor medida las variaciones en los volúmenes de arena. Así se han calculado los volúmenes medios por días de muestreo en la playa alta de los sectores estudiados (Figura 9) derivándose algunos resultados dignos de comentario. La interpretación del volumen sedimentario se puede postular como otro de los paradigmas que incitan a pensar en la importancia que tiene la simbiosis entre factores naturales y antropogénicos en tanto la modificación de la playa. 
Por lo que respeta a causas naturales, hay una fuerte correlación entre la existencia de bermas de restos de $P$. oceanica y los días de mayor volumen. Así, queda claramente reflejado como en los meses invernales, momento en que las bermas vegetales son más presentes y recurrentes, se alcanzan los picos máximos en cuanto a $\mathrm{m}^{3}$ de arena, asumiéndose el máximo en el mes de enero, con un total de $740.930 \mathrm{~m}^{3}$, seguido del mes de marzo, alcanzándose los $715.933 \mathrm{~m}^{3}$. De hecho, si esta información se verifica con la variación anual de la línea de costa (Figura 6), se vuelve a apreciar como las cotas máximas coinciden de nuevo con los días de muestreos tomados en el invierno. En segundo lugar, otro factor natural con fundamento para ser considerado es la presencia de barras sumergidas en el foreshore. De los perfiles tomados se aprecia como las barras arenosas en el mes de enero, y un poco menos en marzo, tienden a estar adheridas a la parte inferior de la playa alta, potenciando a la vez mayores longitudes. A modo de ejemplo, en el caso de enero, de los 6 días muestreados, en 5 se encuentran barras sumergidas, estando éstas adheridas en 4 de ellos.

En cuanto a los mínimos, éstos se localizan en octubre, al final de la temporada estival. No obstante, para una correcta interpretación cabe echar la vista atrás a fin de observar la secuencia que se sigue desde junio. En este periodo de tiempo se aprecia una disminución progresiva, y a la vez considerable, del volumen de sedimento en el backshore, pasando de $594.594 \mathrm{~m}^{3}$ en junio, a $548.779 \mathrm{~m}^{3}$ en agosto, para llegar al mínimo registrado en octubre, con un volumen de $500.066 \mathrm{~m}^{3}$, coincidiendo de lleno con los meses de máxima frecuentación de la playa. El caso de mayo queda un poco aislado, con un volumen que se sitúa en los $572.746 \mathrm{~m}^{3}$, hecho que supone una disminución importante frente a los valores registrados en los meses de enero y marzo. La mediana anual del volumen de arena en la playa alta de Can Picafort y Es Comú de Muro se sitúa en los $612.174 \mathrm{~m}^{3}$.

Si se aborda la caracterización del área para cada uno de los perfiles a nivel individual, y de igual manera que pasaba en tanto a su variabilidad longitudinal, se aprecia una clara diferenciación entre los parámetros que se siguen en la playa urbana y los presentes en la playa natural (Figura 10). En el caso de los perfiles $1\left(438 \mathrm{~m}^{2}\right)$ y 2 (431 $\mathrm{m}^{2}$ ), localizados frente al paseo marítimo, se registran las superficies mínimas, mientras que los valores aumentan si nos situamos en los perfiles del sector natural, con la máxima en el perfil 3, alcanzando los $738 \mathrm{~m}^{2}$. No obstante, el coeficiente de correlación entre la longitud de la playa alta y su área $(r=0,48)$ muestra de correlación positiva aunque moderada, indicando que no siempre una mayor longitud del sector emergido se traduce en una mayor superficie del perfil calculado. A modo de ejemplo el perfil 4 , que con una longitud de playa alta equiparable al perfil 2, su superficie en planta es notablemente superior $\left(209 \mathrm{~m}^{2}\right)$, o bien el perfil 5, que con una longitud de playa alta notablemente inferior al mismo perfil $2(18 \mathrm{~m})$, sus volúmenes son realmente equiparables $\left(\mathrm{P} 5=470,30 \mathrm{~m}^{2}\right.$ vs $\left.\mathrm{P} 2=431,16 \mathrm{~m}^{2}\right)$.

Des del punto de vista geomorfológico se repiten las diferencias entre los perfiles de la playa de Can Picafort y los que se sitúan en Es Comú. El condicionamiento de la playa urbana, sobretodo en temporada alta con una recurrencia diaria, hace que su estado de homogeneización sea mucho más elevado que en el caso del sector más natural, en donde el efecto de la maquinaria para aplanar y limpiar el sustrato no es presente. Así pues, de nuevo se muestran divergencias entre los perfiles 1 y 2 por un lado, y el resto por otro, dibujándose en los dos primeros un perfil mucho más suave y homogéneo que en el resto, sobretodo por lo que representa el sector emergido del sistema (Figura 8). 
Tabla 2

EVIDENCIAS DE TEMPORALES DE MAR EN EL BACKSHORE. DISTANCIAS DESDE EL SWASH $(M)$

\begin{tabular}{|c|c|c|c|c|c|c|}
\cline { 2 - 8 } \multicolumn{1}{c|}{} & Perfil 1 & Perfil 2 & Perfil 3 & Perfil 4 & Perfil 5 & Perfil 6 \\
\hline 13-jun-09 & $/$ & $/$ & $/$ & $/$ & 6 & $/$ \\
\hline 27 -ago-09 & $/$ & $/$ & $/$ & $/$ & 6 & $/$ \\
\hline 27 -oct-09 & 15,5 & 12,5 & 7,5 & 9 & 19,5 & $/$ \\
\hline 30 -ene-10 & 27 & 8 & 18 & $/$ & 4,5 & 9 \\
\hline $27-m a r-10$ & 27 & 10,5 & 21 & 13,5 & $/$ & 9 \\
\hline 29-may-10 & $/$ & $/$ & $/$ & $/$ & $/$ & $/$ \\
\hline Mediana & 23,17 & 10,33 & 15,50 & 11,25 & 9,00 & 9,00 \\
\hline Mediana global & \multicolumn{7}{c|}{$\mathbf{1 3 , 0 4}$} \\
\hline
\end{tabular}

Elaboración propia.

Se aprecian de esta manera cambios de pendiente a lo largo de la playa seca en los perfiles que se localizan en los sectores menos antropizados. La menor frecuentación en la zona y la ausencia de maquinaria de peso a lo largo de la playa, hace que la compactación de sustrato sea sustancialmente inferior, suponiendo al tiempo una mayor vulnerabilidad frente a la fuerza y energía refractada por las olas.

Si bien los patrones que muchas variables nos han mostrado trazan caminos distintos, manifestando a la vez el diferente comportamiento entre la playa urbana y natural, las evidencias de temporales en playa alta se postulan en una misma tendencia. De los registros de campo tomados, queda patente la gran capacidad que los temporales de mar pueden llegar a tener sobre el backshore (Tabla 2). En el caso que nos ocupa, el 70,5\% de los registros tomados frente a temporales de mar se han dado en los meses de octubre, enero y marzo, coincidiendo de lleno con el periodo invernal, a excepción del perfil 5 , en donde también se registraron evidencias en los muestreos de junio y agosto. La capacidad que la fuerza del oleaje tiene para abordar la playa seca es muy variable, no obstante las evidencias máximas advierten de su facilidad para interferir sobre el sector emergido. Los meses de enero y marzo fueron los que mostraron mayores influencias del mar sobre la playa, llegando a los $27 \mathrm{~m}$ tierra adentro en el perfil 1 los días 30 y 27 de los meses respectivos. Por lo que se refiere a las mínimas, y siguiendo la lógica de la hidrodinámica estacional, se dan en los meses de junio y agosto, con evidencias a 6 $\mathrm{m}$ del swash, concretamente en el perfil 5. Si las medianas de los respectivos perfiles no disciernen notablemente de la mediana global, el perfil 1 se muestra como el punto en donde los registros tomados han alcanzado su máxima longitud, indicando con una media de 23,17 m, hasta dónde pueden llegar los parámetros de interacción entre el sector emergido y sumergido de la playa.

\section{Conclusiones}

De los resultados obtenidos pueden derivar dos consideraciones principales. En primer lugar las diferencias en cuanto al comportamiento detectado en la playa urbana de Can Picafort frente al que de da sector natural de Es Comú de Muro. En segunda instancia, la 
variabilidad de las playas se manifiesta de manera dinámica, no sólo en escala temporal, sino que también en una perspectiva espacial. Así pues, la primera consideración de la playa no debe de enfocarse hacia su rendimiento económico sin antes tener presente su condición de espacio dinámico, cambiante y vulnerable. Además de sus características naturales, manifestadas por el comportamiento de todos los agentes ambientales que se dan, la frecuentación humana y la utilización del espacio también tienen voz en cuanto a la variación de su perfil. Las diferencias espaciales determinadas entorno a las dos playas estudiadas suscitan con fundamento que la presión antropogénica tiene un peso de calado en lo que se refiere a las variaciones de perfiles de playa en un periodo de tiempo relativamente corto.

Los cambio de la línea de costa en un período de un año se han mostrado significativos, no obstante con divergencias entre los sectores caracterizados. La variabilidad de la línea de costa en la playa urbana ha sido sustancialmente mayor que la que se ha registrado en la franja más natural. Los patrones de frecuentación por un lado, y las técnicas utilizadas para su limpieza por otro, suscitan una alta correlación en tanto a la modificación de los perfiles de playa y la localización de la zona de swash. Si bien la huella humana debe de considerarse como un agente de primer orden, se determina también la importancia que algunos factores naturales juegan sobre la variación de la cota 0 y la longitud de la playa alta. El mejor ejemplo mostrado son las acumulaciones de restos de P.oceanica, que en temporada invernal provocan un crecimiento del backshore, ganando metros a la zona de swash. Además, se ha confirmado en el periodo de estudio lo que algunos autores ya habían remarcado con anterioridad (Massutí, et al., 2000; San Félix, 2000; Roig-Munar, 2001; Servera, 2004; Roig-Munar, 2004). La retirada masiva en vistas a la temporada turística a través de maquinaria pesada, provoca una aceleración importante frente a la desprotección de la playa, que junto a la alteración provocada por el sobreuso del espacio, supone un retroceso de la línea de costa y una disminución de su espacio utilizable. A parte de las variaciones con una dimensión longitudinal, éstos también varían su forma verticalmente. Las pendientes dibujadas por el trazo de los perfiles disciernen unas de las otras en función de la estación, y también de su localización.

De los cambios longitudinales y verticales deriva también la magnitud de la playa alta en cuanto a su volumen. La variabilidad de pendientes, así como también la energía refractada por el oleaje y corrientes, hacen que los volúmenes de arena vayan manifestando cambios significativos. No obstante, en ese sentido se debe atribuir, de nuevo, una mención especial a las acumulaciones de restos de P. oceanica en la parte inferior del backshore, ya que se manifiesta una importante correlación entre la existencia de estos restos y los días de mayor volumen en la playa alta, en detrimento de los días en donde no existen bermas, coincidiendo con los momentos de menor volumen.

La diferencias entre sectores se manifiestan una vez más si se consideran des del punto de vista morfológico a pequeña escala. No es difícil establecer diferencias entre los perfiles localizados en la playa urbana y los que se sitúan en la franja natural. Sobre todo en temporada alta, momento de máxima frecuentación y explotación de la playa, el acondicionamiento de la playa urbana provoca diferencias morfológicas notables, convirtiendo los perfiles en plataformas planas, aptas para la comodidad del usuario, y eliminando a la vez cualquier alteración geomorfológica. Si bien las diferencias entre el sector más antropizados y la franja perteneciente al sistema playa-duna de Es Comú de Muro han quedado demostrados a través de distintas variables, éstas desaparecen cuando se consideran las evidencias de los temporales de mar sobre la playa seca, que con un $70,5 \%$ de presencia en meses invernales, sigue una distribución considerablemente homogénea a lo largo de las playas estudiadas. 

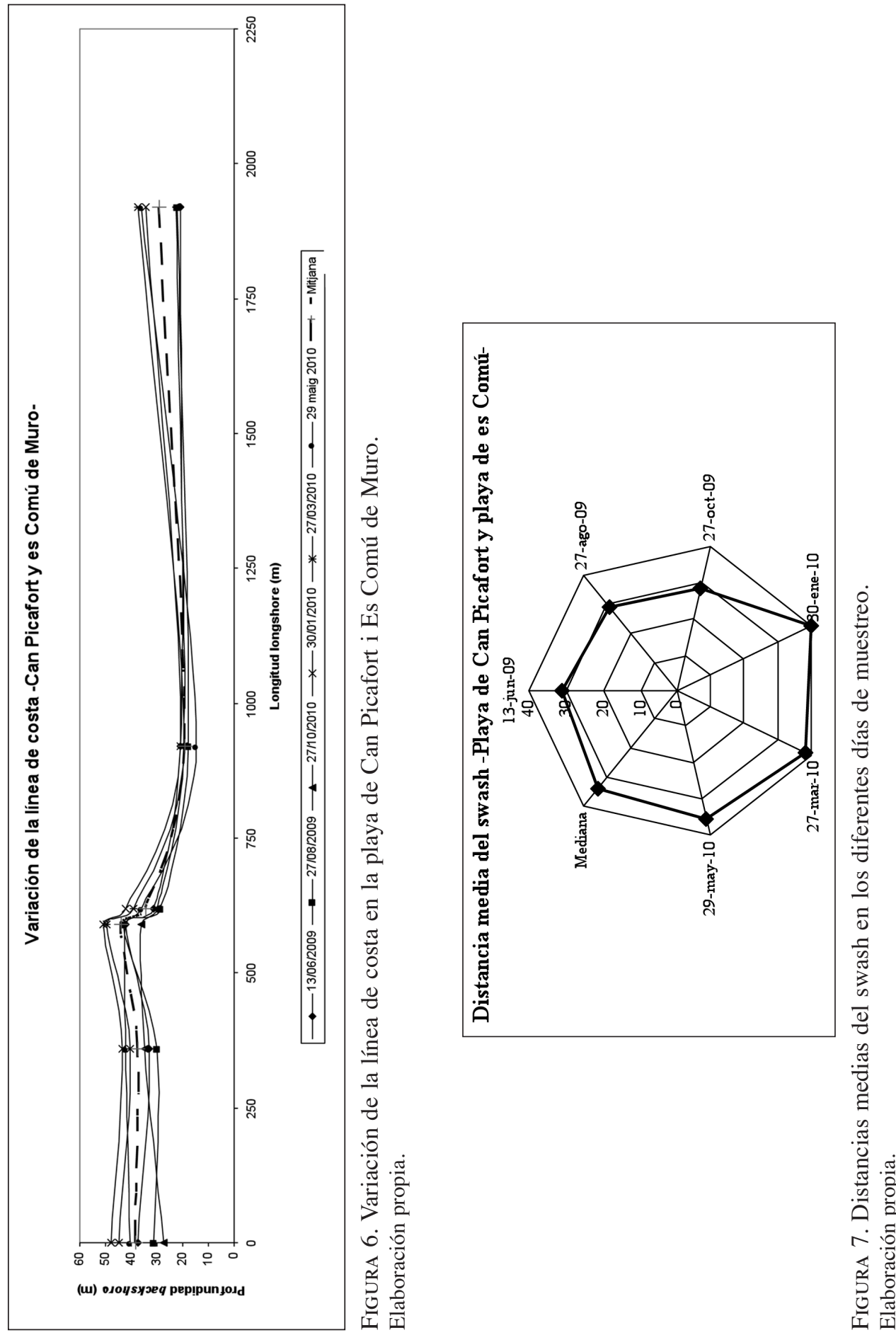

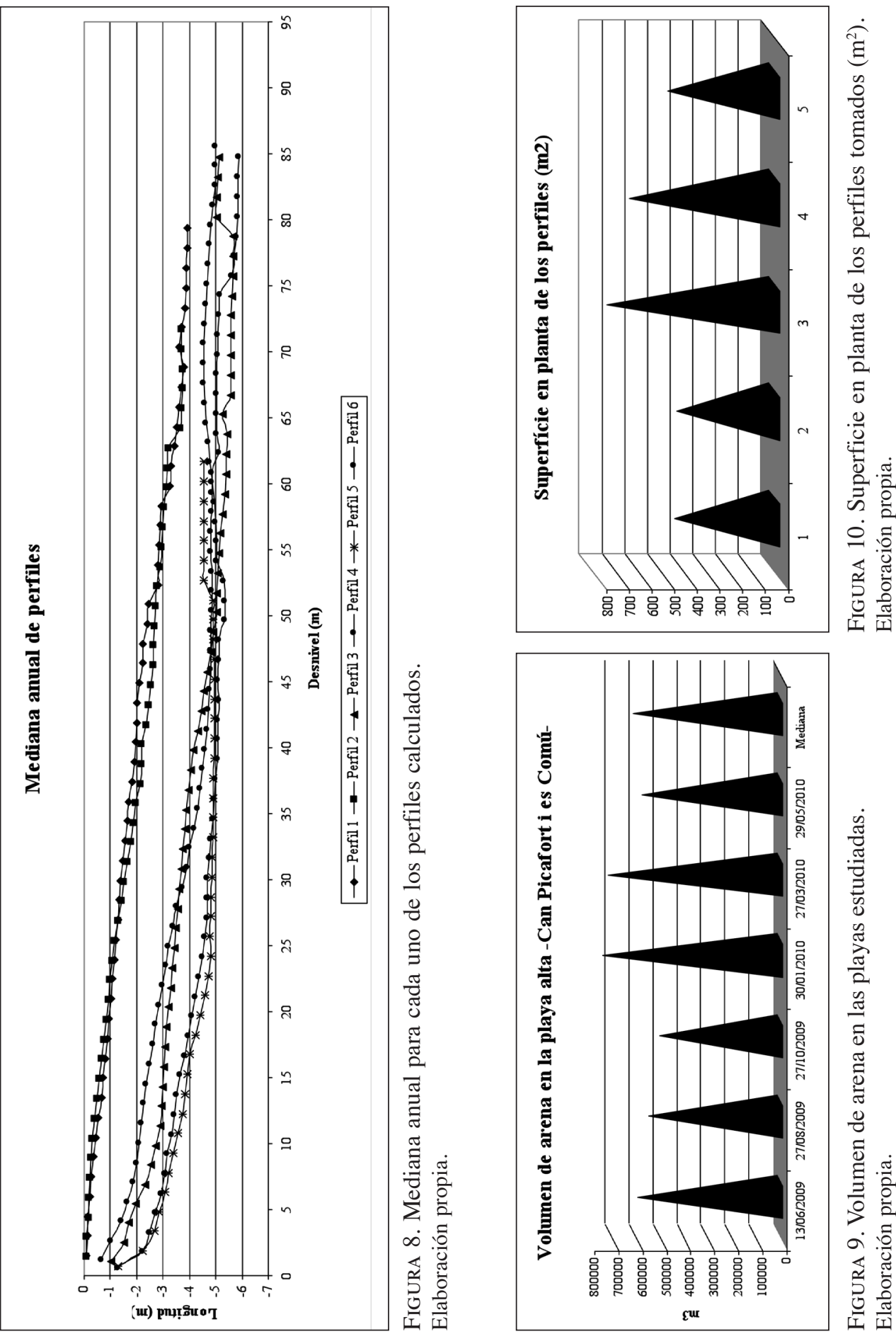
Concluyendo, se consideran cumplidos y demostrados los objetivos principales propuestas en el presente trabajo. Las playas son espacios extremadamente dinámicos, y que su variabilidad no tan sólo se expresa en periodos largos de tiempo, sino que lo hace en una escala temporal mucho más reducida. Esos cambios, ayudados de factores naturales tales como el oleaje o las corrientes litorales, se ven, cuando menos condicionados también, por la acción antrópica sobre las playas.

\section{Bibliografía}

BASTERRETXEA, G., ORFILA, A., JORDI, A., FORNÓS, J., TINTORÉ, J. (2006): «Evaluation of a small volume renourishment strategy on a narrow Mediterranean beach», en Geomorphology, $\mathrm{n}^{\mathrm{o}} 88$, pp. 139-151.

BAUER, B., SHERMAN, J. (1999): «Coastal dune dynamics: Problems and Prospects», en Aeolian enviroments, sediments and landforms. London, Wiley, pp. 71-104.

DELGADO, I., LLOYD, G. (2004): «A simple low cost method for one person beach profiling», en Journal of Coastal Research, $\mathrm{n}^{\circ}$ 20, pp. 1246-1252.

EMERY, K. O. (1961): «A simple method of measuring beach profiles», en Limnology and Oceanography, $\mathrm{n}^{\circ} 6$, pp. 90-93.

GELABERT, B., SERVERA, J., RODRÍGUEZ-PEREA, A. (2002): «Características geomorfológicas del sistema dunar de la Bahía de Alcudia (isla de Mallorca)», en Geogaceta, no 32, pp. 209-212.

GOY, J. L., ZAZO, C., CUERDA, J. (1997): «Evolución de las áreas margino - litorales de la costa de Mallorca (I.Baleares) durante el último y presente interglacial: nivel del mar holoceno y clima», en Boletín Geológico y Minero, $\mathrm{n}^{\mathrm{o}}$ 108, pp. 127-135.

HERNÁNDEZ CALVENTO, L. (2002): Análisis de la evolución del sistema de dunas de Maspalomas, Gran Canaria, Isla de Canarias (1960-2000). Tesis doctoral. Universidad de Las Palmas de Gran Canaria, Gran Canaria.

JUANEDA, J., ROIG-MUNAR, F. X. (2002): «El pla de neteja integral de l'illa de Menorca com a eina de gestió ambiental», en Geografía y territorio, el papel del geógrafo a escala local. Universitat de les Illes Balears, Palma de Mallorca, pp. 43-48.

MARTÍN PRIETO, J. A., SERVERA NICOLAU, J. (2006): «Erosión costera del sector comprendido entre s'Oberta del Gran Canal y el Puerto de Alcúdia (NE. Mallorca)», en Geomorfología y territorio. Actas de la IX reunión nacional de geomorfología. Santiago de Compostela, pp. 433-442.

MAS FORNERS, A. (1992): «Expansión agraria, turismo y desagrarización en Mallorca durante el siglo XX. Una aportación a partir del estudio de una comunidad rural (Inédito»). Comunicación presentada en el Segundo Congreso de Jóvenes Geógrafos e Historiadores, Sevilla.

MASSUTÍ, E., GRAU-JOFRE, A., DUARTE, C., TERRADOS, J., MARBÀ, N. (2000): La posidònia: l'alga que no és. Conselleria d'Agricultura i Pesca ,Palma de Mallorca, 59 pp.

MURRAY, I. (2005): «Huellas en la playa de s'Arenal. La huella del impacto humano sobre la T(t) ierra y en las Islas Baleares», en Sociedad y medio ambiente. Actas de las II Jornadas Sociedad y medio ambiente. Salamanca, pp. 197-250.

NONN, H. (1987): Geografía de los litorales. Akal, Madrid, 199 pp.

OCDE (1995): Gestión de zonas costeras. Políticas integradas. Ediciones Mundiprensa Madrid.

PULEO, J. A., PEARRE, N. S., HE, L., LAUREN, S., O'NEAL, M., PIETRO, L. S., FOWLER, M. (2008): «A single-user subaerial beach profiler», en Journal of Coastal Research, $\mathrm{n}^{\circ}$ 24, pp. 1080-1086.

ROIG-MUNAR, F. X. (2001): «El conocimiento de la Posidonia oceanica y sus funciones ecológicas como herramienta de gestión litoral. La realización de encuestas a los usuarios de playas y calas de la isla de Menorca», en Papeles de Geografía, n 34, pp. 271-280.

ROIG-MUNAR, F. X. (2003): «Identificación de variables útiles para la clasificación y gestión de playas y calas. El caso de la isla de Menorca (I. Baleares)», en Boletín de la A.G.E., no 35, pp. 175-190.

ROIG-MUNAR, F. X. (2004): «Análisis y consecuencias de la modificación artificial del perfil de playa-duna provocado por el efecto mecánico de su limpieza», en Investigaciones Geográficas, no 33 , pp. 87-103. 
ROIG-MUNAR, F. X., COMAS LAMARCA, E. (2005): «Propuestas de un modelo de clasificación para las playas de las Islas Baleares mediante el análisis de variables de uso, estado y gestión», en Boletín de la A.G.E., $\mathrm{n}^{\circ}$ 40, pp. 429-448.

SAN FELIX, M. (2000): La posidònia. El bosc submergit. Edicions Documenta Balear. Palma de Mallorca, pp. 77.

SCHÜRGERS, P. (2007): Diagnóstico espacial del estado turístico. El núcleo turístico de Can Picafort en el municipio de Santa Margalida (Inédito). Düsseldorf.

SERVERA, J. (1997): Els sistemes dunars litorals de les Illes Balears. Tesis doctoral. Departament de Ciències de la Terra, Universitat de les Illes Balears. Palma de Mallorca, pp. 904.

SERVERA, J. (2002): «Eurosion Case Study. Can Picafort, Isle of Mallorca, Spain», en http://www. eurosion.org/shoreline/47 canpicafort.html

SERVERA, J. (2004): Geomorfologia del Litoral de les Illes Balears. Edicions Documenta Balear. Palma de Mallorca.

SUAREZ DE VIVERO, J. L. (1999): «Delimitación y definición del espacio litoral», en Actas de las Jornadas sobre el litoral de Almería: caracterización, ordenación y gestión de un espacio geográfico. Almería, pp. 13-23.

YEPES, V. (2002): «La explotación de playas. La madurez del sector turístico», en Ingeniería y territorio, $\mathrm{n}^{\circ} 61, \mathrm{pp} .72-77$. 
